\title{
Angular Accuracy of ML, MUSIC, ROOT-MUSIC and spatially smoothed version of MUSIC algorithms
}

\author{
M. Jalali, B. Honarvar Shakibaei, Department of Electrical Engineering, IAU, Urmia, Iran
}

\begin{abstract}
This article first discusses the simulation of ML, MUSIC, ROOT-MIUSIC and spatially Smoothed base MUSIC and then compares the algorithms based on their estimation of the accuracy level angels from two different sources, having demonstrated the result of simulation of the four algorithms in comparison tables, it is observed that ROOT-MUSIC algorithm have high level of accuracy in estimation and recognition of close angles. MUSIC algorithm and Spatially Smoothed version of MUSIC algorithm come next.
\end{abstract}

Index Terms-DOA, ML, MUSIC, Root MUSIC, Spatially Smoothed algorithms.

\section{INTRODUCTION}

Several algorithms have been introduced and used to estimate Direction Of Arrival (DOA) and the most basic ones are Capon Maximum Likelihood (ML)[1] and MUSIC algorithm introduced by Dr. Schmitt[2]. The numerous researches done in this field have improved MUSIC algorithm which led to the ROOT-MUSIC and Spatially Smoothed version of MUSIC [4]. ROOT-MUSIC algorithm creates polynomials and determines its root and estimates the angles and solves the deficiency of MUSIC algorithm to detect in spaces related to estimation parameters. The three algorithms i.e. ML, MUSIC and ROOT-MUSIC are efficient only when the signals are non-coherent, and will not be efficient if the arrival signals are coherent. Spatially Smoothed version of MUSIC improves and modifies preprocessing covariance matrix by dividing arrays to sub arrays and presents a good performance the presence of coherent signals.

In the present study, in addition to the presence of the capon's ML algorithm, MUSIC, ROOT-MUSIC and Spatially Smoothed version of MUSIC in the second section, the relations and the equations used for both methods are given.

In the third section, considering the findings of the other articles and the simulations done in the present research, the number of sensors, the snapshots needed to be taken from a signals, the ratio of signals to noise ratio (SNR) and appropriative distance between array elements and based on that, in the rest of this section, the estimation of the arrival

Manuscript received June 28, 2009, and accepted August 13, 2009. This work was supported in part by the I.A.U Department of Electrical Engineering.

M. Jalali is with the Islamic Azad University of Urmia Branch, Iran phone: +98914-389-9112; e-mail: jalali.mahdi@gmail.com).

B. Honarvar Shakibaei is with the Islamic Azad University of Urmia Branch,Iran(phone:+989143471440;email:barmak.honarvar@gmail.com). angles for four algorithms in the presence of two sources i.e. $28^{\circ}$ and $30^{\circ}$ has been carried out [9]. For evaluation of the accuracy level of the angles of the simulation of the four algorithms by calculating average of the estimated angles, deviation from the mean, variance of the angles and the number of successful experiments are presented. In the fourth section, called conclusion, the best way for estimation and clarify of the angles based on the accuracy level is introduced.

\section{DIRECTION-OF- ARRIVAl ESTIMATION AlgORITHMS}

\section{A. Capon's ML Algorithm}

This method is one of the simplest techniques foe DOA estimation. Figure 1 shows the classical narrowband beamformer structure, where the output signal $\mathrm{y}(\mathrm{k})$ is given by a linearly weighted sum of the sensor element outputs. That is

$$
y(k)=w^{H} u(k)
$$

The total output power of the conventional can be expressed as

$$
\begin{aligned}
P_{c b f}= & E\left[|y(k)|^{2}\right]=E\left[\left|w^{H} u(k)\right|^{2}\right]= \\
& w^{H} E\left[u(k) u^{H}(k)\right] w=w^{H} R_{u u} w
\end{aligned}
$$

Where $R_{u u}$ is the autocorrelation matrix of the array input data. Consider a signal $s(k)$ impinging on the array at the angle an $\phi_{0}$. The power at the beamformer output can be expressed as:

$$
\begin{aligned}
& P_{c b f}\left(\phi_{0}\right)=E\left[\left|w^{H} u(k)\right|^{2}\right]=E\left[\left|w^{H}\left(a\left(\phi_{0}\right) s(k)+n(k)\right)\right|^{2}\right]= \\
& \quad\left(\left|w^{H} a\left(\phi_{0}\right)\right|^{2}\left(\sigma_{s}^{2}+\sigma_{n}^{2}\right)\right)
\end{aligned}
$$

Where $a\left(\phi_{0}\right)$ is the steering vector associated with the DOA angle $\phi_{0}, n(k)$ is the noise vector at the array input, and $\sigma_{s}^{2}=E\left[s^{2}(k)\right]$ and $\sigma_{n}^{2}=E\left[n^{2}(k)\right]$ are the signal 


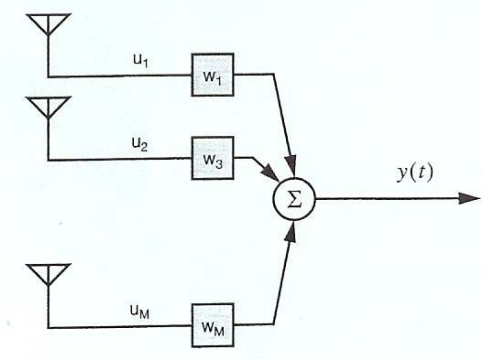

Figure 1. Illustration of the classical beamforming structure

Power and noise power, respectively. It is clearly seen from (3) that the output power is maximized when $w=$ $a\left(\phi_{0}\right)$.this is because $w=a\left(\phi_{0}\right)$ aligns the phases of the signal components arriving from $\phi_{0}$ at the sensors, causing them to add constructively. The output power at the classical beamformer as a function of the Angle-Of-Arrival is given by

$$
P_{c b f}(\phi)=w^{H} R_{u u} w=a^{H}(\phi) R_{u u} a(\phi)
$$

Capon's ML method uses some (not all) of the degrees of freedom to form a beam in the desired look direction while simultaneously using the remaining degrees of freedom to form nulls in the direction of interfering signals. This technique minimizes the contribution of the undesired interferences by minimizing the output power while maintaining the gain along the look direction to be constant, usually unity. That is,

$$
\begin{array}{r}
\min _{w} E\left[|y(k)|^{2}\right]=\min _{w} w^{H} R_{u u} w \\
\text { Subject to } \quad w^{H} a\left(\phi_{0}\right)=1
\end{array}
$$

Using Lagrange multiplier, the weight vector that solves (5) can be shown to be

$$
w=\frac{R_{u u}^{-1} a(\phi)}{a^{H}(\phi) R_{u u}^{-1} a(\phi)}
$$

The output power of the array as a function of the AngleOf-Arrival, using Capon's method, is given by Capon' spatial spectrum,

$$
P_{\text {Capon }}(\phi)=\frac{1}{a^{H}(\phi) R_{u u}^{-1} a(\phi)}
$$

By computing and plotting Capon's spectrum over the whole range of $\phi$, the DOA's can be estimated by locating the peaks in the spectrum.

\section{B. MUSIC Algorithm}

The MUSIC algorithm that presented first time by Schmidt [3] has been used more in recent years. MUSIC algorithm can only be used to estimate incoherent signal sources. For coherent signals, the performance of MUSIC will be degraded and it is not an efficient tool. Recent studies shows MUSIC can approximately provide a solution of multipath fading; also multipath fading causes similar receiving signals in chip length in some systems like CDMA. $\alpha\left(\theta_{m}\right)$ is the array response (or steering) vector, corresponding to the DOA of the $m^{\text {th }}$ signal, and is defined as:

$$
\alpha\left(\theta_{m}\right)=\left[\exp \left(-j(n-1) 2 \pi\left(\frac{d}{\lambda}\right) \sin \left(\theta_{m}\right)\right)\right]^{T},
$$

Where ${ }^{T}$ is the transpose operator, and $\lambda$ is the wavelength of the incident signals. The combination of all possible steering vectors forms the array manifold matrix A.

The MUSIC algorithm starts by applying temporal averaging over $\mathrm{K}$ snapshots (or samples) taken from the leads to forming a spatial correlation (or covariance) matrix $\mathrm{R}$ defined as:

$$
R=\frac{1}{K} \sum_{t=1}^{K} x(t) x^{H}(t)
$$

Where ${ }^{H}$ denotes the Hermitian operator. Substituting $x(t)$ from (8) into (9) results in:

$$
\begin{gathered}
R=\frac{1}{K} \sum_{t=1}^{K} A(\theta) s(t) s^{H}(t) A^{H}(\theta)+n(t) n^{H}(t) \\
R=A R_{S S} A^{H}+\sigma_{n}^{2} I
\end{gathered}
$$

Where $R_{S S}$ the signal covariance matrix is $\sigma_{n}^{2}$ is the noise variance and $I$ is an identity matrix of size $N \times N$.

In the MUSIC algorithm, the eigenvectors $E_{n}$ of matrix R that correspond to the smallest eigenvalues from the noise subspace. The eigenvectors $E_{n}$ and the steering vectors that make up matrix $A$ are used to form the MUSIC angular spectrum which is given by:

$$
P(\theta)=\frac{A^{H}(\theta) A(\theta)}{A^{H}(\theta) E_{n} E_{n}^{H} A(\theta)}
$$

The orthogonality between the eigenvectors gives rise to peaks in the MUSIC angular spectrum. These peaks correspond to the directions of arrival of the signals impinging on the sensor array. It is well known that with an $\mathrm{N}$-element sensor array, MUSIC can detect up to N-1 uncorrelated signals [9].

\section{ROOT-MUSIC Algorithm}

For the case of a uniformly spaced linear array with interelement spacing $\mathrm{d}$, the $\mathrm{m}^{\text {th }}$ element of the steering vector $a(\phi)$ may be expressed as:

$$
\begin{gathered}
a_{m}(\phi)=\exp \left(j 2 \pi m\left(\frac{d}{\lambda}\right) \cos (\phi)\right) \\
m=1, \ldots, M
\end{gathered}
$$

The MUSIC spectrum is an all-pole function of the form

$$
P_{\text {MUSIC }}(\theta)=\frac{1}{a^{H}(\phi) V_{n} V_{n}^{H} a(\phi)}=\frac{1}{a^{H}(\phi) C a(\phi)}
$$

Where $C=V_{n} V_{n}^{H}$. using equation (14) may be written as 


$$
P_{M U S I C}^{-1}=\sum_{m=1}^{M} \sum_{n=1}^{M} \exp \left(-j \frac{2 \pi m d}{\lambda} \cos \phi\right) C_{m n} \exp \left(j \frac{2 \pi m d}{\lambda} \cos \phi\right)
$$

Where $C_{m n}$ is the entry in the $m^{\text {th }}$ row and $n^{\text {th }}$ column of $C$. Combining the two summations into one, (15) can be simplified as

$$
P_{M U S I C}^{-1}=\sum_{n=1}^{M} C_{l} \exp \left(j \frac{2 \pi d}{\lambda} l \cos \phi\right)
$$

Where $C_{l}=\sum_{m-n=l} C_{m n}$ is the sum of the entries of $C$ along the $l^{\text {th }}$ diagonal.

By defining a polynomial $D(z)$ as follows,

$$
D(z)=\sum_{l=-M+1}^{M+1} C_{l} z^{-1}
$$

Evaluating the MUSIC spectrum $P_{\text {MUSIC }}(\phi)$ becomes equivalent to the polynomial $D(z)$ on the unit circle, and the peaks in the MUSIC Spectrum exist because the roots of $D(z)$ lie close to the unit circle. Ideally, with no noise, the poles will lie in exactly on the unit circle at locations determined by the DOA. In other words, a pole of $D(z)$ at $z=z_{1}=\left|z_{1}\right| \exp \left(j \arg \left(z_{1}\right)\right)$ will result in a peak in the MUSIC spectrum at $\cos \phi=\left(\frac{\lambda}{2 \pi d}\right) \arg \left(z_{l}\right)$.

\section{D. spatially smoothed music algorithm}

The signal covariance matrix $R_{S S}$ is a full-rank matrix (i.e., non-singular) as long as the incident signals on the sensor array are uncorrelated, which is the key to the MUSIC eigenvalues decomposition. However, if the incident signals become highly correlated, which is a realistic assumption in practical radio environments, matrix $R_{S S}$ will lose its non-singularity property and, consequently, the performance of MUSIC will degrade severely. In this case, spatial smoothing (SS) must be used to remove the correlation between the incident signals by dividing the main sensor array into forward/backward overlapping subarrays and introducing phase shifts between these sub-arrays.

The vector of the received signals at the $k^{\text {th }}$ forward subarray is expressed as:

$$
x_{k}^{F}(t)=A D^{(k-1)} s(t)+n_{k}(t)
$$

Where $(k-1)$ denotes the $k^{\text {th }}$ power of the diagonal matrix $D$ given by:

$$
D=\operatorname{diag}\left\{e^{-j \frac{2 \pi}{\lambda} \sin \theta_{1}}, \ldots, e^{-j \frac{2 \pi}{\lambda} \sin \theta_{m}}\right\}
$$

The spatial correlation matrix $R$ of the sensor array is then defined as the sample mean of the covariance matrices of the forward sub-arrays:

$$
R=\frac{1}{L} \sum_{k=0}^{L-1} R_{k}^{F}
$$

Here $L$ is the number of overlapping sub-arrays. When applying FSS, the N-elements sensor array can detect up to N/2 correlated signals [7], [9] .

\section{Simulations of The Algorithms}

\section{A. Assumptions for simulation}

All simulation processes in the presented study are carried out using MATLAB software considering the following conditions:

- Uniformed linear array (ULA), consisting of 16 sensors and distance between the elements equals half of the wave length.

- The number of snapshots taken from a signal (N-channel) equals 1000.

- The ratio of signal to noise (SNR) equals $20 \mathrm{~dB}$ for two uncorrelated sources when the angles are $28^{\circ}$ and $30^{\circ}$.

- The mean is calculated for 1000 independent experiment of mont carlo.

- In spatially smoothed version of MUSIC, two coherent signals with different ranges, from two paths with equal powers and a delay time shorter than the length of a chip in WCDMA system are applied.

- The length of the sub-array (L) in spatially smoothed algorithm equals 5[9].

\section{B. Simulation results}

Figure (2) indicates the diagram derived from the four algorithms for two sources at $28^{\circ}$ and $30^{\circ}$.

In figure (a), spectrum of Capon's ML algorithm is presented and it is deserved that this algorithm has a lower level of accuracy. This method determines the degree of freedom for forming the pattern in the desired look direction and simultaneously uses the remaining degree of freedom to form nulls in the direction of interfering signals. Though this method is not efficient for distinguishing two adjacent angles. Figure (b) presents the finding based on the application of MUSIC algorithm for estimation of two different angles. This algorithm, which is based on subspace principles, approximately solves that problem caused by accuracy level of the previous algorithm. Figure (c) indicates the findings of ROOT-MUSIC. Figure (d) presents the findings of spatially smoothed algorithm. For this algorithm, the angles of two sources are $28^{\circ}$ and $30^{\circ}$, but these two sources unlike the three before mentioned algorithms, are completely correlative and the delay time between two signals is shorter than the length of a chip. The accuracy level of MUSIC, ROOT-MUSIC and spatially smoothed version of MUSIC is approximately same for these two angles. For a better evaluation and comparison of the findings, estimated angles, standard deviation and the variance of the angles are given. 


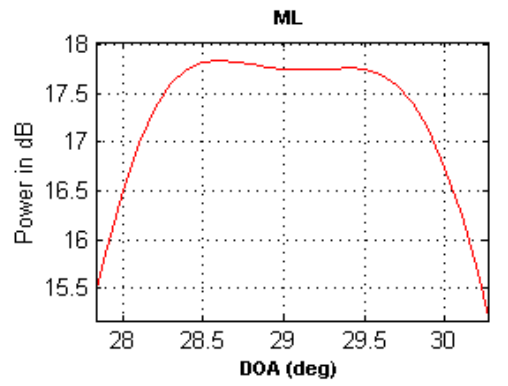

(a)

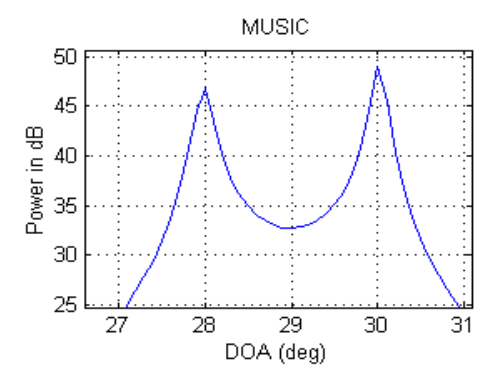

(b)

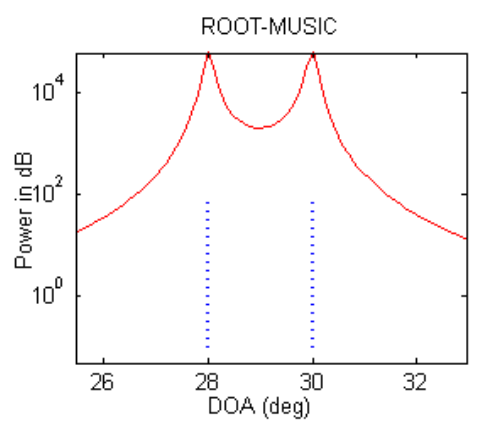

(c)

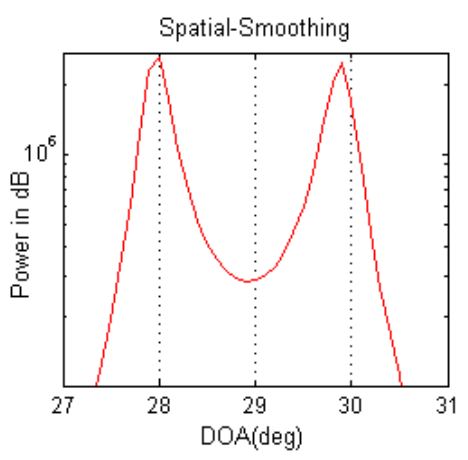

(d)

Figure 2 . Estimation of two angles $\left(28^{\circ}\right.$ and $\left.30^{\circ}\right)$ with four algorithms

C. Evaluation of the estimation level of the algorithms

Comparison between the findings of figures (1) and (2) shows that ML algorithm dose not present an acceptable estimation of the array for $28^{\circ}$ and $30^{\circ}$ and it works when the angle increases gradually to $28.2^{\circ}$, but considering the instances of the percentage of the successful experiments mean, deviation from the mean and the variance of the angles, the accuracy of the algorithm is very low and it is not an appropriate algorithm for the estimation of two adjacent angles. However, MUSIC algorithm provides acceptable results for $29.4^{\circ}$, not being efficient for above that. Tables (3) and (4) indicate the findings of ROOTMUSIC algorithm and spatially smoothed version of MUSIC.

Undoubtedly, ROOT-MUSIC algorithm provides the most acceptable results of all and even can estimate up to $29.85^{\circ}$ and has the highest level of accuracy.

TABLE 1. ACCuracy Amount of ML Algorithm In Distinguish Two ADJACENT ANGLES

\begin{tabular}{|c|c|r|r|r|}
\hline Angles & $\begin{array}{l}\text { Percent } \\
\text { age of } \\
\text { Successful } \\
\text { Iterations }\end{array}$ & $\begin{array}{r}\text { Dstimation } \\
\text { average }\end{array}$ & $\begin{array}{l}\text { deviation } \\
\text { from the } \\
\text { mean }\end{array}$ & $\begin{array}{l}\text { Variance } \\
\text { of } \\
\text { Angels }\end{array}$ \\
\hline$\theta_{1}=28$ & 100 & 28.3785 & -0.3785 & 0.0033 \\
$\theta_{2}=30$ & & 29.6130 & 0.3870 & 0.0025 \\
\hline$\theta_{1}=28.1$ & 86 & 28.5820 & -0.4820 & 0.0053 \\
$\theta_{2}=30$ & & 20.5163 & 0.4837 & 0.0045 \\
\hline$\theta_{1}=28.2$ & 14.5 & 28.8103 & -0.6103 & 0.0060 \\
$\theta_{2}=30$ & & 29.3966 & 0.6034 & 0.0046 \\
\hline
\end{tabular}

TABLE 2. ACCURACY AMOUNT OF MUSIC ALGORITHM IN DISTINGUISH

\begin{tabular}{|c|c|c|c|c|}
\hline \multicolumn{1}{|c|}{ TWO ADJACENT ANGLES } \\
& $\begin{array}{c}\text { Percent } \\
\text { age of } \\
\text { Success } \\
\text { ful } \\
\text { Iteration } \\
\mathrm{s}\end{array}$ & $\begin{array}{c}\text { DOA } \\
\text { Estimation } \\
\text { average }\end{array}$ & $\begin{array}{c}\text { deviatio } \\
\text { from the } \\
\text { mean }\end{array}$ & $\begin{array}{c}\text { Variance } \\
\text { of } \\
\text { Angels }\end{array}$ \\
\hline$\theta_{1}=28$ & 100 & 28.0005 & $-0 . .0005$ & 0.0001 \\
$\theta_{2}=30$ & & 29.9985 & 0.0015 & 0.0001 \\
\hline$\theta_{1}=28.2$ & 86 & 28.5820 & -0.4820 & 0.0053 \\
$\theta_{2}=30$ & & 20.5163 & 0.4837 & 0.0045 \\
\hline$\theta_{1}=29$ & 100 & 28.0005 & $-0 . .0005$ & 0.0001 \\
$\theta_{2}=30$ & & 29.9985 & 0.0015 & 0.0001 \\
\hline$\theta_{1}=29.2$ & 89.5 & 29.2827 & -0.0827 & 0.0050 \\
$\theta_{2}=30$ & & 29.9045 & 0.0955 & 0.0040 \\
\hline$\theta_{1}=29.3$ & 45 & 29.3978 & -0.0978 & 0.0049 \\
$\theta_{2}=30$ & & 29.8944 & 0.1056 & 0.0041 \\
\hline$\theta_{1}=29.4$ & 7.5 & 29.5067 & -0.1067 & 0.0092 \\
$\theta_{2}=30$ & & 29.8867 & 0.1133 & 0.0069 \\
\hline
\end{tabular}

TABLE 3. ACCURACY AMOUNT OF ROOT-MUSIC ALGORITHM IN DISTINGUISH TwO ADJACENT ANGLES

\begin{tabular}{|c|c|c|c|c|}
\hline Angles & $\begin{array}{c}\text { Percentage } \\
\text { of } \\
\text { Successful } \\
\text { Iteration }\end{array}$ & $\begin{array}{c}\text { Estimatio } \\
\mathrm{n} \\
\text { averag } \\
\mathrm{e}\end{array}$ & $\begin{array}{c}\text { deviation } \\
\text { from the } \\
\text { mean }\end{array}$ & $\begin{array}{c}\text { Variance } \\
\text { of } \\
\text { Angels }\end{array}$ \\
\hline$\theta_{1}=28$ & 100 & 27.999 & 0.0002 & 0.1525 \\
$\theta_{2}=30$ & & 8 & 0.0003 & $\times 10^{-3}$ \\
& & 29.999 & & 0.3479 \\
& & 7 & & $\times 10^{-3}$ \\
\hline$\theta_{1}=28.6$ & 100 & 28.608 & - & 0.0006 \\
$\theta_{2}=30$ & & 29.998 & 0.0008 & 0.0007 \\
\hline$\theta_{1}=29$ & 100 & 29.003 & - & 0.0014 \\
$\theta_{2}=30$ & & 0 & 0.0030 & 0.0013 \\
& & 29.998 & 0.0020 & \\
\hline$\theta_{1}=29.2$ & 100 & 29.282 & - & 0.0050 \\
$\theta_{2}=30$ & & 7 & 0.0827 & 0.0040 \\
& & 29.904 & 0.0955 & \\
\hline$\theta_{1}=29.4$ & 100 & 29.430 & - & 0.0047 \\
$\theta_{2}=30$ & & 3 & 0.0303 & 0.0052 \\
& & 29.966 & 0.0338 & \\
\hline
\end{tabular}




\begin{tabular}{|c|c|c|c|c|}
\hline & & 2 & & \\
\hline$\theta_{1}=29.6$ & 100 & 29.684 & - & 0.0209 \\
$\theta_{2}=30$ & & 8 & 0.0848 & 0.0253 \\
& & 29.941 & 0.0587 & \\
& & 3 & & \\
\hline$\theta_{1}=29.85$ & 94 & 29.663 & - & 0.1525 \\
$\theta_{2}=30$ & & 6 & 0.1864 & 0.2099 \\
& & 30.251 & - & \\
& & 0 & 0.2510 & \\
\hline
\end{tabular}

TABLE 4. ACCURACY AMOUNT OF SPATIALly SMOOTHED ALGORITHM IN

\begin{tabular}{|c|c|c|l|l|}
\hline \multicolumn{5}{|c|}{ AISTINGUISH TWO ADJACENT ANGLE } \\
\hline & $\begin{array}{l}\text { Percentage } \\
\text { of } \\
\text { Successful } \\
\text { Iterations }\end{array}$ & $\begin{array}{c}\text { DOA } \\
\text { Estimation } \\
\text { average }\end{array}$ & $\begin{array}{l}\text { deviation } \\
\text { from the } \\
\text { mean }\end{array}$ & $\begin{array}{l}\text { Variance of } \\
\text { Angels }\end{array}$ \\
\hline$\theta_{1}=28$ & 99.6 & 28.0040 & -0.0040 & 0.0009 \\
$\theta_{2}=30$ & & 29.9898 & 0.0102 & 0.0067 \\
\hline$\theta_{1}=28.2$ & 96.2 & 28.1587 & 0.0413 & 0.0065 \\
$\theta_{2}=30$ & & 29.9603 & 0.0397 & 0.0063 \\
\hline$\theta_{1}=28.4$ & 90.4 & 28.4201 & -0.0201 & 0.0073 \\
$\theta_{2}=30$ & & 29.8763 & 0.1237 & 0.0089 \\
\hline$\theta_{1}=28.6$ & 84 & 28.3986 & 0.2014 & 0.0237 \\
$\theta_{2}=30$ & & 29.8231 & 0.1769 & 0.0103 \\
\hline$\theta_{1}=28.8$ & 20.6 & 28.9146 & -0.1146 & 0.0018 \\
$\theta_{2}=30$ & & 29.7951 & 0.2049 & 0.0383 \\
\hline & & & & \\
\hline
\end{tabular}

\section{CONCLUSION}

In the present article, four important algorithms, i.e. Capon's ML, MUSIC, ROOT-MUSIC and spatially smoothed version of MUSIC for two users in the environment including noise and fading, are simulated and the accuracy level of them for estimation of the angles close to each other is evaluated. The findings in the tables and figures indicate that the highest amount of accuracy is for music and ROOT-MUSIC respectively. The lowest level of accuracy is Capon's ML algorithm. The low level of accuracy for spatially smoothed version of MUSIC is due to natural processes of the calculation of the mean for this method.

\section{REFERENCES}

[1] J. Capon, "High Resolution Frequency Wave Number Spectrum Analysis", Proceedings of IEEE, Vol. 57, pp. 1408-1418, August 1969.

[2] R. O. Schmidt, "Multiple Emitter Location and Signal Parameter Estimation", IEEE Transactions on Antenna and Propagation, Vol. AP-34, pp.276-280, 1986.

[3] B. D. Rao and K. V. Hari, "Performance Analysis of ROOT-MUSIC", IEEE Transactions on ASSP, Vol. ASSP-37(12), pp.1939-1949, December 1989

[4] T. J. Shan, M. Wax and T. Kailath, "On Spatial Smoothing for Direction Of Arrival Estimation of Coherent Signals", IEEE Transactions on ASSP-33, No. 4, August 1985.

[5] F. A. Belhoud, R. M. Shubair and M. E. Al-Mualla, "Modeling and Performance Analysis of DOA Estimation Adaptive Signal Processing Arrays", IEEE, ETISALAT, ICECS-2003.

[6] R. S. Kawitkar and D. G. Wakde, "An Approach for MUSIC Algorithm in Smart Antenna System", IEEE International Workshop on Antenna Technology, IEEE 2005.
[7] E. M. Al-Ardi, R. M. Shubair and M. E. Al-Mualla, "Computationally Efficient DOA Estimation in a Multipath Environment using Covariance Differencing and Iterative Spatial Smoothing", IEEE 2006.

[8] W. Shilong and L. Jingqing, "DOA Estimation of Correlative Signals with Spatial Smoothing Technique and Modified Music Method", ICSP Proceedings of IEEE, pp. 1411-1414, 2004.

[9] M.Jalali and B.Honarvar Shakibaei, "Performance Evaluation of Spatially Smoothed MUSIC (Considering Fading and Both Coherent and Non-Coherent Signals)" GESTS 1414, International Transaction on computer Science and Engineering, Vol.47,Num.1,June 2008.

Mehdi Jalali was born in Urmia, Iran, in 1980. He received his B.S degree in telecommunication engineering from Department of Electrical Engineering, Urmia Azad University in 2003 and M.S. degree from Department of Electrical Engineering, Tehran Azad University in 2006. Since 2006, he has taught telecommunication courses in Urmia Azad University. His research interests include electromagnetic fields.

Barmak Honarvar Shakibaei was born in Urmia, Iran, in 1973.He received his B.S. degree in telecommunication engineering from Department of Electrical Engineering, Urmia Azad University in 1995 and M.S. degree from Department of Electrical Engineering, Tabriz University in 1998. Since 1998, he is an academic staff in Urmia Azad University. His research interests include DSP, digital audio filter design and power systems. 